\title{
Erratum
}

\section{Multiplicative rule of Schubert classes ${ }^{\star}$}

\section{Haibao Duan ${ }^{1}$, Xuezhi Zhao ${ }^{2}$}

1 Institute of Mathematics, Chinese Academy of Sciences, Beijing 100080, P.R. China (e-mail: dhb@math.ac.cn)

2 Department of Mathematics, Capital Normal University, Beijing 100037, P.R. China (e-mail: zhaoxve@mail.cnu.edu.cn)

Oblatum 2-I-2006 \&

Published online: 21 September 2006 - (C) Springer-Verlag 2006

Invent. math. (2004) Digital Object Identifier (DOI) 10.1007/s00222-004-0394-Z

Published online: 2. September 2004 - (C) Springer-Verlag 2004

The correct statement of [D, Lemma 4.1] should be as follows.

Lemma 4.1. The Kronecker paring $H^{2}(G / T) \times H_{2}(G / T) \rightarrow \mathbb{Z}$ can be expressed in term of Cartan numbers as

$$
\left\langle e_{\gamma}, \varphi_{\beta *}[S(y ; \beta)]\right\rangle=\gamma \circ \beta, \beta, \gamma \in \Phi,
$$

where $[S(y ; \beta)] \in H^{2}(S(y ; \beta))(=\mathbb{Z})$ is the orientation class.

Consequently, in [D, Definition 1], the Cartan matrix $A_{w}=\left(a_{i, j}\right)$ of $w$ associated to the decomposition (2.1) should be given by

$$
a_{i, j}= \begin{cases}0 & \text { if } i \geq j \\ -\beta_{j} \circ \beta_{i} & \text { if } i<j\end{cases}
$$

and the statement (2) of [D, Lemma 4.5] should be changed to

the structure matrix of $S\left(y ; \beta_{1}, \cdots, \beta_{k}\right)$ (with respect to $x_{1}, \cdots, x_{k}$ ) is $A=\left(a_{i j}\right)_{k \times k}$, where

$$
a_{i j}= \begin{cases}0 & \text { if } i \geq j \\ -\beta_{j} \circ \beta_{i} & \text { if } i<j .\end{cases}
$$

* The online version of the original article can be found at http://dx.doi.org/10.1007/s00222-004-0394-z 
Based on [D, Theorem] a program calculating the Chow rings of flag varieties were composed [DZ]. We realized the error while testing the program for some simple cases: the Chow rings of $B_{3} / T$ and $C_{3} / T$ were switched.

This error takes no effect when computing with flag varieties of the types $A_{n}, D_{n}, E_{6}, E_{7}, E_{8}$ since their Cartan matrices are all symmetric. In contrast the Cartan matrices of $B_{n}$ and $C_{n}$ differ by transposition.

\section{References}

[D] Duan, H.: Multiplicative rule of Schubert classes. Invent. Math. 159, 407-436 (2005)

[DZ] Duan, H., Zhao, X.: The Chow rings of generalized Grassmannians. arXiv: math.AG/0511332 